\title{
Cristal and Azurite: new tools for integration-by-parts reductions
}

\author{
Alessandro Georgoudis \\ Department of Physics and Astronomy, Uppsala University, SE-75108 Uppsala, Sweden \\ E-mail: Alessandro.Georgoudis@physics.uu.se
}

Kasper J. Larsen*

School of Physics and Astronomy, University of Southampton, Highfield, Southampton, SO17 1BJ, United Kingdom

E-mail: Kasper.Larsen@soton.ac.uk

\section{Yang Zhang}

PRISMA Cluster of Excellence, Johannes Gutenberg University, 55128 Mainz, Germany

E-mail: zhang@uni-mainz.de

Scattering amplitudes computed at a fixed loop order, along with any other object computed in perturbative quantum field theory, can be expressed as a linear combination of a finite basis of loop integrals. To compute loop amplitudes in practice, such a basis of integrals must be determined. We discuss AZURITE (A ZURich-bred method for finding master InTEgrals), a publicly available package for finding bases of loop integrals. We also discuss CRISTAL (Complete Reduction of IntegralS Through All Loops), a future package that produces the complete integration-by-parts reductions.

13th International Symposium on Radiative Corrections (Applications of Quantum Field Theory to Phenomenology)

25-29 September, 2017

St. Gilgen, Austria

${ }^{*}$ Speaker. 


\section{Introduction}

Precision calculations of the cross sections of Standard Model processes at the Large Hadron Collider are crucial to gain a quantitative understanding of the background and in turn improve the ability to extract signals of new physics. This typically requires computations at next-to-nextto leading order (NNLO) in fixed-order perturbation theory, in order to match the experimental precision and the parton distribution function uncertainties.

A key tool in these calculations are integration-by-parts (IBP) identities [1-15]. These are relations that arise from the vanishing integration of total derivatives,

$$
\int \prod_{j=1}^{L}\left(\frac{\mathrm{d}^{D} \ell_{j}}{\mathrm{i} \pi^{D / 2}}\right) \sum_{i=1}^{L} \frac{\partial}{\partial \ell_{i}^{\mu}} \frac{v_{i}^{\mu}}{D_{1}^{\alpha_{1}} \cdots D_{k}^{\alpha_{k}}}=0
$$

where the vectors $v_{i}^{\mu}$ are polynomials in the internal and external momenta, the $D_{k}$ denote inverse propagators, and the $\alpha_{i} \geq 1$ are integers. The IBP identities allow the loop integrals that contribute to a loop-level quantity, say a scattering amplitude, to be expressed in a finite basis of integrals. ${ }^{1}$ In practice, this leads to a very significant simplification of the representation of the amplitude. IBP reductions moreover allow setting up differential equations for the basis integrals, thereby enabling their evaluation [17-22].

An initial step of generating IBP reductions is to determine a basis of integrals. In this proceedings contribution we discuss the SINGULAR [23]/MathematiCA package AZURITE (A ZURichbred method for finding master InTEgrals) [24] which determines a basis for the space of integrals spanned by a given $L$-loop diagram and all of its subdiagrams (obtained by pinching lines). As the underlying algorithm is completely general, AZURITE can be used to provide a basis for any number of loops and external particles, arbitrary configurations of internal and external masses in both the planar and non-planar sectors. In practice, the running time for two-loop diagrams is typically of the order of a few seconds, and for three-loop diagrams a few minutes at most. AzURITE has been tested up to five loops.

Related work has appeared in ref. [25] where the number of basis integrals is determined as the sum of the Milnor invariant evaluated at the critical points of the polynomials that enter the parametric representation. This method has moreover been implemented in the MATHEMATICA package Mint.

\section{Integration-by-parts identities on maximal cuts}

We start by setting up notation and conventions. We consider an $L$-loop integral with $k$ propagators and $m-k$ irreducible scalar products (i.e., polynomials in the loop momenta and external momenta which cannot be expressed as a linear combination of the inverse propagators). We work in dimensional regularization and normalize the integral as follows,

$$
I\left(\alpha_{1}, \ldots, \alpha_{m} ; D\right) \equiv \int \prod_{j=1}^{L} \frac{\mathrm{d}^{D} \ell_{j}}{\mathrm{i} \pi^{D / 2}} \frac{D_{k+1}^{\alpha_{k+1}} \cdots D_{m}^{\alpha_{m}}}{D_{1}^{\alpha_{1}} \cdots D_{k}^{\alpha_{k}}} \quad \text { with } \quad \alpha_{i} \geq 0 .
$$

\footnotetext{
${ }^{1}$ The fact that the basis of integrals is always finite was proven in ref. [16].
} 
AZURITE determines integration-by-parts identities (1.1) on unitarity cuts, where some set of propagators are put on shell, $D_{i}=0$ for $i \in \mathscr{S}$. To this end we make use of the Baikov representation [26] whose variables are the inverse propagators and the irreducible scalar products, $z_{\alpha} \equiv D_{\alpha}$ where $1 \leq \alpha \leq m$. In order to write down the associated Jacobian, we define the set of independent external and loop momenta, $\left\{v_{1}, \ldots, v_{n+L-1}\right\}=\left\{p_{1}, \ldots, p_{n-1}, \ell_{1}, \ldots, \ell_{L}\right\}$ and consider the associated Gram determinant, $F=\operatorname{det}_{i, j=1, \ldots, n+L-1}\left(v_{i} \cdot v_{j}\right)$. We can now express the integral in eq. (2.1) in its Baikov representation,

$$
I(\alpha ; D) \propto \int \mathrm{d} z_{1} \cdots \mathrm{d} z_{m} \frac{z_{k+1}^{\alpha_{k+1}} \cdots z_{m}^{\alpha_{m}}}{z_{1}^{\alpha_{1}} \cdots z_{k}^{\alpha_{k}}} F(z)^{\frac{D-L-n}{2}} .
$$

To find integration-by-parts identities on the maximal cut $z_{1}=\cdots=z_{k}=0$ of this diagram, we consider the most general total derivative of the same form as the residue of eq. (2.2) at this pole,

$$
\begin{aligned}
0 & =\int \frac{\mathrm{d} z_{k+1} \cdots \mathrm{d} z_{m}}{z_{k+1}^{-\alpha_{k+1}} \cdots z_{m}^{-\alpha_{m}}} \sum_{i=k+1}^{m} \frac{\partial}{\partial z_{i}}\left(a_{i}(z) F(z)^{\frac{D-n-L}{2}}\right) \\
& =\int \frac{\mathrm{d} z_{k+1} \cdots \mathrm{d} z_{m}}{z_{k+1}^{-\alpha_{k+1}} \cdots z_{m}^{-\alpha_{m}}} \sum_{i=k+1}^{m}\left(\frac{\partial a_{i}}{\partial z_{i}}+\frac{D-n-L}{2 F(z)} a_{i}(z) \frac{\partial F}{\partial z_{i}}\right) F(z)^{\frac{D-n-L}{2}}
\end{aligned}
$$

where the $a_{i}(z)$ denote polynomials. We observe that, for an arbitrary choice of $a_{i}(z)$, the terms in the parenthesis ( $\cdots)$ in eq. (2.4) correspond to integrals in $D$ and $D-2$ dimensions, respectively. This is because the $\frac{1}{F(z)}$ factor in the second term effectively modifies the integration measure, shifting the space-time dimension from $D$ to $D-2$.

We can avoid the dimension shift by choosing the $a_{i}(z)$ such that,

$$
\sum_{i=k+1}^{m} a_{i}(z) \frac{\partial F}{\partial z_{i}}+b F=0
$$

where $b$ denotes a polynomial, since then the $\frac{1}{F}$ factor in eq. (2.4) cancels out, and the ansatz in eq. (2.3) corresponds to an integration-by-parts identity in purely $D$ dimensions.

Equations of the form (2.5) are known in algebraic geometry as syzygy equations. Algorithms for obtaining a generating set of solutions are known and have been implemented in several computer algebra systems dedicated to computational algebraic geometry, such as Singular [23] and Macaulay2 [27]. By plugging the obtained set of solutions of eq. (2.5) into eq. (2.4), we then find the required integration-by-parts identities evaluated on the maximal cut $z_{1}=\cdots=z_{k}=0$.

\section{Algorithm of AZURITE}

The algorithm of AZURITE can be summarized as follows. Given an input set of inverse propagators $D_{1}, \ldots, D_{k}$ and irreducible numerator insertions $D_{k+1}, \ldots, D_{m}$, AZURITE determines a basis of integrals by proceeding through the following steps.

1. Find the corresponding graph $\Gamma$ and the automorphism groups of $\Gamma$ and all of its subgraphs.

2. Find a list $\mathscr{C}$ of cuts such that no two elements of $\mathscr{C}$ are related by any of the symmetries found in step 1 . 
3. For each cut $c \in \mathscr{C}$, construct IBP identities and symmetry relations on $c$, giving $\mathbb{Z}_{p}$ values to external kinematical invariants and the space-time dimension $D$ for efficiency.

4. For each cut $c \in \mathscr{C}$, apply Gauss-Jordan elimination to the system of symmetry relations and IBP identities found in step 3. The resulting non-pivot entries correspond to basis integrals.

In the current implementation of AZURITE, step 1 is realized by graph theory functions in MATHEMATICA. The construction of IBP identities on maximal cuts in step 3 is carried out with the formalism explained in section 2. In setting up the linear systems to which Gauss-Jordan elimination is applied in step 4, AzURITE sorts the integrals in descending order by the following order relation. For two integrals $I(\alpha ; D)$ and $I(\beta ; D)$ with propagator powers $\alpha=\left(\alpha_{1}, \ldots, \alpha_{k}\right)$ and $\beta=\left(\beta_{1}, \ldots, \beta_{k}\right)$, we have $I(\alpha ; D)>I(\beta ; D)$ if $\sum_{i=1}^{k}\left|\alpha_{i}\right|>\sum_{i=1}^{k}\left|\beta_{i}\right|$ or, in the event of a tie, $\sum_{i=k+1}^{m}\left|\alpha_{i}\right|>\sum_{i=k+1}^{m}\left|\beta_{i}\right|$ or, in the event of a further tie, $\left(\alpha_{1}, \ldots, \alpha_{m}\right)>\left(\beta_{1}, \ldots, \beta_{m}\right)$ lexicographically. To improve efficiency, AzURITE makes use of two graph-based simplifications: 1) diagrams with massless tadpoles are detected and discarded; and 2) on any given cut $c$, rather than using the parent momenta of the top-level graph, AZURITE uses the available momenta (which may be sums of several parent momenta). This reduces the number of scalar products.

\section{Performance of AZURITE}

The figure below shows the computation time (on a single core on a standard laptop) and number of basis integrals for a variety of diagrams at various loop orders and configurations of internal and external masses.

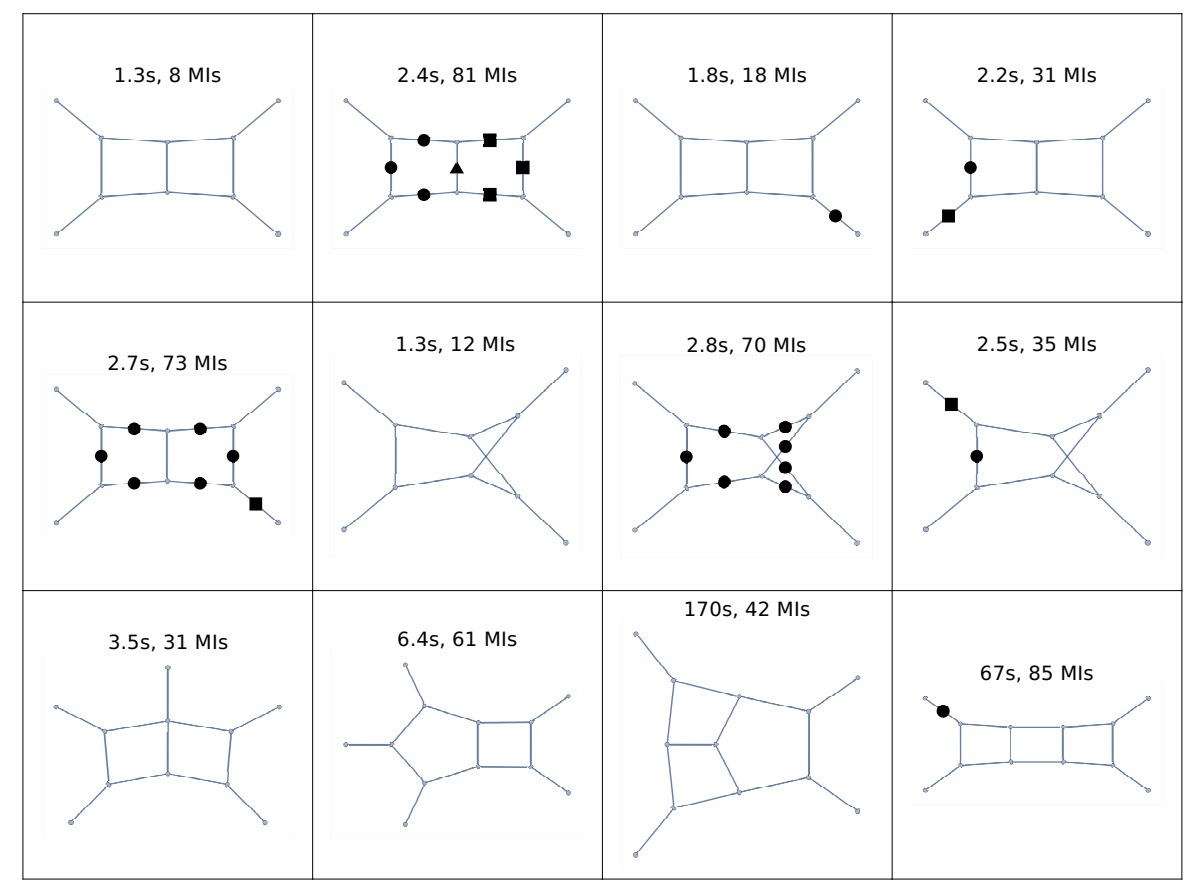

Figure 1: Computation time and number of basis integrals for different topologies and mass configurations. Here, $\boldsymbol{\Lambda}, \mathbf{\square}$ and $\bullet$ represent different masses. 
In a new version, AZURITE 2 [28], to be made publicly available shortly, the computation time is reduced significantly, particularly for three- and higher-loop topologies. An example is provided in fig. 2.

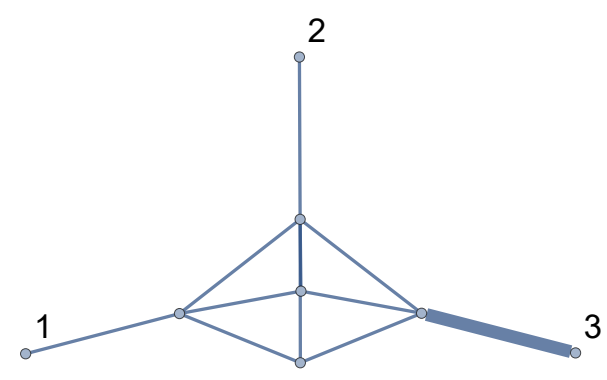

Figure 2: Integral topology for the four-loop form factor. For this diagram, the computation time to determine an integral basis is reduced from $180 \mathrm{~s}$ in version 1 of AZURITE to $41 \mathrm{~s}$ in version 2.

\section{CRISTAL}

CRISTAL (Complete Reduction of IntegralS Through All Loops) is a future package that will produce the complete integration-by-parts reductions. It is based on the formalism developed in ref. [15]. The basic idea is to study the integration-by-parts identities (1.1) on a spanning set of cuts, defined as the maximal cuts of those elements of an integral basis $\mathscr{B}$ which cannot be obtained from another $b \in \mathscr{B}$ by adding propagators,

$$
\mathscr{C}=\{c \in \mathscr{B}: \nexists b \in \mathscr{B}: \quad b \text { is a strict subgraph of } c\} .
$$

The effect of constructing the IBP reductions on the set of cuts $\mathscr{C}$ is, roughly speaking, to blockdiagonalize the linear systems to which Gauss-Jordan elimination is applied in the standard Laporta algorithm. We emphasize that a spanning set of cuts (5.1) can only be determined once an integral basis $\mathscr{B}$ is known. Thus, AZURITE provides an initial step for the full reduction problem undertaken by CRISTAL.
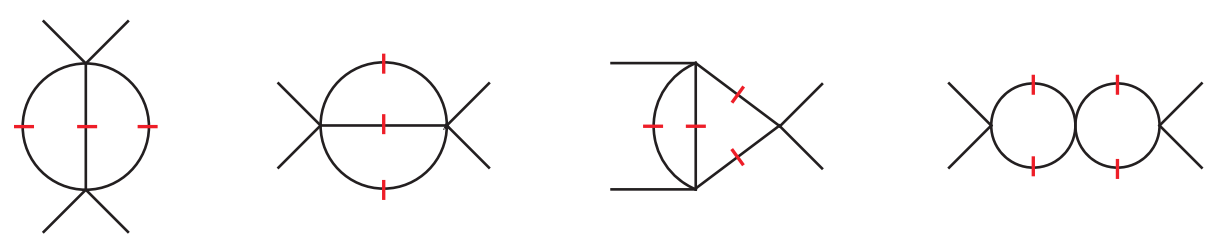

Figure 3: Complete IBP reductions can be efficiently obtained by studying the IBP identities on a spanning set of cuts, defined in eq. (5.1). 


\section{References}

[1] F. Tkachov, Phys.Lett. B100, 65 (1981).

[2] K. Chetyrkin and F. Tkachov, Nucl.Phys. B192, 159 (1981).

[3] S. Laporta, Phys. Lett. B504, 188 (2001), hep-ph/0102032.

[4] S. Laporta, Int.J.Mod.Phys. A15, 5087 (2000), hep-ph/0102033.

[5] V. A. Smirnov and M. Steinhauser, Nucl. Phys. B672, 199 (2003), hep-ph/0307088.

[6] C. Anastasiou and A. Lazopoulos, JHEP 0407, 046 (2004), hep-ph/0404258.

[7] A. Smirnov, JHEP 0810, 107 (2008), 0807.3243.

[8] C. Studerus, Comput.Phys.Commun. 181, 1293 (2010), 0912.2546.

[9] J. Gluza, K. Kajda, and D. A. Kosower, Phys.Rev. D83, 045012 (2011), 1009.0472.

[10] A. von Manteuffel and C. Studerus, (2012), 1201.4330.

[11] R. N. Lee, (2012), 1212.2685.

[12] A. von Manteuffel and R. M. Schabinger, Phys. Lett. B744, 101 (2015), 1406.4513.

[13] A. V. Smirnov, Comput. Phys. Commun. 189, 182 (2015), 1408.2372.

[14] H. Ita, Phys. Rev. D94, 116015 (2016), 1510.05626.

[15] K. J. Larsen and Y. Zhang, Phys. Rev. D93, 041701 (2016), 1511.01071.

[16] A. V. Smirnov and A. V. Petukhov, Lett. Math. Phys. 97, 37 (2011), 1004.4199.

[17] A. V. Kotikov, Phys. Lett. B254, 158 (1991).

[18] A. V. Kotikov, Phys. Lett. B267, 123 (1991), [Erratum: Phys. Lett.B295,409(1992)].

[19] Z. Bern, L. J. Dixon, and D. A. Kosower, Nucl. Phys. B412, 751 (1994), hep-ph/9306240.

[20] E. Remiddi, Nuovo Cim. A110, 1435 (1997), hep-th/9711188.

[21] T. Gehrmann and E. Remiddi, Nucl.Phys. B580, 485 (2000), hep-ph/9912329.

[22] J. M. Henn, Phys. Rev. Lett. 110, 251601 (2013), 1304.1806.

[23] W. Decker, G.-M. Greuel, G. Pfister, and H. Schönemann, Singular 4-0-2 - A computer algebra system for polynomial computations, http://www.singular.uni-kl.de, 2015.

[24] A. Georgoudis, K. J. Larsen, and Y. Zhang, Comput. Phys. Commun. 221, 203 (2017), 1612.04252 . 
[25] R. N. Lee and A. A. Pomeransky, JHEP 11, 165 (2013), 1308.6676.

[26] P. A. Baikov, Phys. Lett. B385, 404 (1996), hep-ph/9603267.

[27] D. R. Grayson and M. E. Stillman, Macaulay2, a software system for research in algebraic geometry, Available at http://www.math.uiuc.edu/Macaulay2/.

[28] A. Georgoudis, K. J. Larsen, and Y. Zhang, (to appear). 